\title{
Second baseman defense techniques in a double play with runner on first
}

\author{
Haonan Yuan \\ Tianjin University Renai College, Tianjin 30163, china \\ 58482477@qq.com
}

Keywords: First base manned; Second baseman; Double play; Defensive technique

\begin{abstract}
In the baseball game, the second is a key base for runner. The second baseman plays a key role in the game. In addition to his ability possess other fielders, he must be sensitive, light, fast, and steady. The most important thing is to keep head clear and clearly understand the situation on the field. This article finds out the relevant information of second baseman's double play technique by searching a large number of documents. complete each step of the technical movement in the most reasonable and fastest way, and use some basic theoretical links to practice to effectively highlight the value of the second baseman completing the double play in the game.
\end{abstract}

\section{Introduction}

In the baseball game, the second is a key base for runner. If you can enter the second base, you have a great hope of scoring. Therefore, the second is generally called the score base. The second baseman plays a key role in the game. In addition to his ability possess other fielders, he must be sensitive, light, fast, and steady. The most important thing is to keep head clear and clearly understand the situation on the field. In a baseball game, an infielder received a ground ball and passed it to the second baseman to block the runnerwho runs from the first base to the second base, then the second baseman or the shortstop caught the ball,touched the base, threw the ball to the first base to stop runner. This process is called double play. Doubleplay is a key role in the game. After a double play is completed, the morale of the whole team can be encouraged, so that the spirit of the team can be invigorated and confident. At the same time, the self-confidence of the opposing team can also be reduced. Complete each step of the technical movement in the most reasonable and fastest way, and use some basic theoretical links to practice to effectively highlight the value of the second baseman completing the double play in the game.

\section{The research method}

2.1 literature data method: to find out the relevant information of second baseman's double play technique by searching a large number of documents.

2.2 Observation Method: Through the watching of live games and video recording, concluded the second baseman's match in the games.

\section{Results and analysis}

3.1 the second baseman's defensive position when someone at the first base

The second baseman has three angles in the defense: depth, medium, and shallow. Regardless of the defensive position, must see both the batter and the pitcher. In the game, the second baseman can choose the defensive position according to the hitting habit of the batter. But when not knowing the hitting habit, the second baseman can see the pitching signal given by the pitcher. According to the pitching line, the defensive position is favorable for quick completion of the double play after catching the ball. In addition, the second baseman can also geta chance to start beforehand by observing the batter's bat and gripping hands.

3.2 Several double play methods which second baseman caught and passed ballwhen someone at the first base 
After the batter hit the ground, the second baseman caught and passed the ball. No matter how good the second baseman's foot moves, and how agile his movements are, if he can't catch the ground ball, he can't be referred to as a excellentsecond baseman. To complete the doubleplaycatching is the most important, while the ground ball is fast, slow, left, right, bouncing high, low, and there are several types of regular and irregular jumping. Therefore, when catching the ground ball, it is necessary to watch the movement. Then judging the rolling direction of the ball, the body is pressed down towards the ball, on the front of the ball, knees bent, then the ball caught on the right hand. Received. In the double play match, the second baseman chooses a reasonable passing method according to the direction of the incoming ball and the position after the ball is received, and the strength of the passing ball is grasped according to different passing ways. Differentground rolling balls have different directions and different double play method.

3.2.1 Double play when a second baseman is facing fast ground ball

When the batter hits quickly roll the ballforward to the second base, the second baseman catches the ball, and pass to the shortstop who has entered the second base parcel blocks the runner who runs from the firstto the second. Thenthe shortstop quickly passes to first to block the runners. Completethe double play. This double play method requires the second baseman to catch the ball steady, fast action, pass the ball accurately. When the second baseman catches the ball from the front, he catches the ball in a low position in front of the ball. When the ball is caught, the left heel and the right toe to beon a same line. The ball is in the middle, but he does not stand up after receiving the ball and turns to the left and knees to throw the ball. Throwing to the shortstop with $50 \%$ strength avoid error, which make shortstop cannot receive the ball and can't complete the double play. If you are close to the baseline when you catch the ball, your left foot will have to move forward and away again, so that you can easily turn around.

\subsubsection{Double play when a second baseman is facing slow ground ball}

The second baseman rushes forward when catching the ball, while running, takes the left foot forward when catching the ball, and sends the ball to the side already in two with the lower body position in the turn. The baseman's shortstop banned the runner who ran from first base to second, then quickly passed by the shortstop to first to block the runner to finish the double play. This kind of double play is the hardest. The second baseman needs to catch the ball with running and throw with turning around. This requires the second baseman to have a solid foundation and a good sense of direction.

3.2.3 Double playwhen a batter hits the ball forward the left side of the second baseman

When the batter hits the ball in the direction of the first base,the second baseman uses the counterclockwise method to catch the ball and turn around to the left,throw the ball to the shortstop who has entered the second and blocks to runner from the first base. Then quickly passed by the shortstop to the first baseman to block the runner to finish the double play. This method requires the second baseman to turn large and stretch, so that the center of gravity can be stabilized; the left foot must point to the second base, and the shortstop must see the left or right side of the second base. Because it is a bit farther from the base of the second base when it receives the ball near the first base. It needs to use $80 \%$ of the power to throw the ball to the shortstop,so that take the time to complete the double play.

3.2.4Double play when a batter hits the ball forward the right side of the second baseman

When the batter hits the ball on the right side of the second baseman, the second baseman will use the backhand to throw the ball after moving to catch the ball. This method is required to be completed in the process of moving, to run side by side while throwing, donot pause. When faced with the second base, the ball is thrown with one hand instead of his hands. After both hands are behind the glove, throw the ball to the shortstop with one hand. Another way is to save time and use the backhand to catch the ball when the ball exceeds the second base. When the ball is received, throw the ball directly to the shortstop with gloves. When doing these two kinds of throwing balls, they are closer to the shortstops. They only need to gently throw them to the shortstops and do not require a hard pass.

3.2.5 Double play when is filled by second baseman with a runner on first 
When the batter hits a ground ball in the direction of between first and second base (close to the first base defensive position), the first baseman throw ball to the shortstop who has entered the second base, and the second baseman run to the first base at the same time as the first baseman catches the ball, catching the ballwhich from the shortstop to complete the double play. This method requires the second baseman to quickly run to the first place and accurately receive the ball from the shortstop.

3.3 Double play when the second baseman two catch and two throwwith a runner on first

Two catch and two throwmeans the shortstop and third baseman catch the ball which the batter hits the ground ball, and throwit to the second baseman. The second baseman catches and passes the ball. When a batter hits a ball forward third base or shortstop, the third baseman or shortstop catches the ball and then throws to the second baseman who has entered the second base. After the second baseman catches the ball, the first runs to the second. Then quickly turned to the first base to block the runners to finish the double play. This method requires the second baseman to respond quickly after the batter hits the ball and advances to the second base in order to prepare for double play. In the case where no or one out of the runner and has a base, the batter will try his best to get the runner to second base, while the defensive one must strive to achieve double play. Sometimes the offensive and defensive results will determine the outcome of the game. When the second baseman reaches the second base, there are different double play method based on different passes from the third baseman and the shortstop.

3.3.1 Double play method when the third baseman catches the ball and throws it to the second baseman

When the third base catches the ball and throws it to the second baseman,should crosses the second base by waiting, because when a ball forward to the third base, you have enough time to cross the second base and wait for the ball. The distance from the third baseman to the second baseman to catch the ball is shorter. Take a step toward the first base before catching the ball, touch the base with the toe of the right foot and quickly throw it to the first base to complete the double play.

3.3.2 Double play method of the second baseman when the shortstop catchesa ball near the second base

When a shortstop catches a ground ball near the second base pack, left foot touch base. Because the distance from the shortstop to the second base is closer, there is no time to wait for the ball. So using the left foot touch the baseby catching the ball from the second. When the ball is caught, the body leans back and moves the center of gravity to the right foot. The left foot moves toward the first base to quickly pass the ball to the first base to complete double play.

\section{Conclusion}

With the development of international baseball, Chinese baseball is gradually on the right track. Baseball technology has also gradually improved, requiring players to practice basic skills assiduously, so that the second baseman must master all aspects of the defensive double play technique with a runner on first, it must be selected best defensive position according to the different conditions of the batter. When the batter hits the infielder's different defensive positions, he can skillfully master the different double play methods; at the same time, he can skillfully grasp the throw when the batter hits the second base. The way of the ball and the power of the throw; but also skillfully mastering the double play method of the second baseman on the base when other infielders catch the ball and pass it to the second base. These techniques require more practice in peacetime training. I hope my research will make some contribution to Chinese baseball.

\section{References:}

[1] Wu Xiangmu, Strong Baseball Theory [M]. Taiwan Provincial Sports Magazine

[2] Meng Fanlin, Wang Shuhong, Lu Bing, Baseball [M] Shuhai Publishing House 
[3] Baseball Technology Graphics [M] People's Sports Press

[4] Qiu Huiqun, Yan Moxiang, Li Baojun, [M] People's Sports Press

[5] Peace, Li Zonghao, Olympic baseball softball [M] People's Sports Press 